\title{
Midterm Results of Transaxillary Occluder Device Closure of Perimembranous Ventricular Septal Defect Guided Solely by Transesophageal Echocardiography
}

\author{
Weijie Liang, MD, ${ }^{1}$ Sijie Zhou, MD,${ }^{1}$ Taibing Fan, MD, PHD,${ }^{1}$ Shubo Song, MD,${ }^{1}$ Bin Li, MD,${ }^{1}$ \\ Haoju Dong, MD,${ }^{1}$ Linhong Zhang, ${ }^{1}$ Manman Hu, MD, ${ }^{1}$ Lin Liu, MD, PHD ${ }^{2}$ \\ ${ }^{1}$ Children's Heart Center, People's Hospital of Zhengzhou University, Zhengzhou, China; ${ }^{2}$ Department of Ultra-sound, People's \\ Hospital of Zhengzhou University, Zhengzhou, China
}

\section{ABSTRACT}

Background: Perimembranous ventricular septal defect (pmVSD) is a common congenital heart disease. Transaxillary occluder device closure of the pmVSD has been proved effective and an alternative to surgical closure. The study aimed to evaluate the immediate operation outcomes and the early and midterm follow-up outcomes of transaxillary occluder device closure.

Methods: From January 2014 to December 2017, we retrospectively analyzed the patients who underwent transaxillary device closure of the pmVSD. All patients underwent transthoracic echocardiography (TTE), chest x-ray, and electrocardiogram (ECG) before and after the procedure (before discharging). Follow-up evaluation was completed at the time of 3, 6, 12 months and yearly thereafter in outpatient setting with TTE and ECG.

Results: A total of 428 patients (216 male, 212 female) underwent transaxillary occluder device closure of the pmVSD under the guidance of transesophageal echocardiography (TEE). The mean age at the operation time was $2.2 \pm 1.5$ year (range 0.5-16.2 year). The mean weight was $8.5 \pm 4.1 \mathrm{~kg}$ (range 6-61 kg). The mean size of the occluder implanted in the operation was $5.3 \pm 1.4$ (range $4-8 \mathrm{~mm}$ ), matching the mean defect size of $4.2 \pm 1.1$ (range 3-6 mm). The device closure operation was successfully achieved in 422 pmVSD patients (98.6\%), and 6 patients failed in occluding and were converted to open surgery because of a great residual shunt and obvious device-related aortic regurgitation . Immediate complete closure was detected by postoperative TEE in all, but 3 patients had trivial residual shunting. Total early adverse events emerged in 47 patients $(11.1 \%)$. New mild tricuspid and aortic regurgitation occurred in 17 and 3 patients and disappeared in follow-up. Abnormal atrioventricular conduction events emerged in 23 patients, including left anterior block, complete right bundle branch block (CRBBB), incomplete right bundle branch block (IRBBB),

Received August 25, 2018; received in revised form November 15, 2018; accepted December 4, 2018.

W.L. and S.Z. contributed equally to this work.

Correspondence: Dr. Taibing Fan, MD, PHD, Department of Cardiovascular Surgery, People's Hospital of Zhengzhou University, 7\# Wei Wu Road, Zhengzhou, Henan Province, 450000, P.R. China (e-mail:fantaibing@163.com). administrated with close follow-up. Pericardial effusion occurred in 2 other patients, managed with puncture drainage. During a median follow-up period of 26.8 months (range 6-48 months), no serious adverse event and later-on complete atrioventricular block were encountered.

Conclusion: In our experience, transaxillary device closure was performed via right infra-axillary mini-incision (invisible) guided by TEE, with low incidence of postoperative adverse events, confirming that transaxillary device closure of the pmVSD under the guidance of TEE is an effective alternative to surgical closure in well-selected pmVSD patients.

\section{INTRODUCTION}

Congenital heart disease (CHD), as one of the lethal birth defects, mostly causing morbidity and mortality of $\sim 0.8 \%$ live-born [Bouma 2017]. Ventricular septal defects (VSDs) are a common type of CHD with a high incidence. If left untreated, severe morbidity and mortality may develop with or without an evident symptom for most of the time.

With advances in surgical managements and skills, open heart surgical repairment has been considered the golden standard treatment for closing a VSD, having achieved excellent outcomes till now[Green 1965; Hufnagel 1965; Hong 2018,]. However, postoperative cardiopulmonary bypass (CPB) complications and incision scars come along with its good results. Percutaneous transcatheter closure of perimembranous ventricular septal defect (pmVSD) developed, avoiding CPB and incision scar [Patel 2009; Polat 2016; Narin 2018]. Despite the obvious advantage of its minimal effect, the potential damage of radiation and contrast agents, especially for young patients, even complete atrioventricular block (AVB), could bring patients to bad situations [Bai 2015; Karaçelik 2015; Ghosh 2017]. After combining the merits of surgery and the internal transcatheter, minimally invasive periventricular device closure of the pmVSD appeared [Xing 2009; Ou-Yang 2015; Song 2017] and was widespread fast when initial experience proved its safety and feasibility.

In this study's technique, distinguishable from traditional transthoracic device closure, we performed the technique via a right infra-axillary approach, an invisible incision. Here, we report our initially clinical experience and midterm follow-up results. 


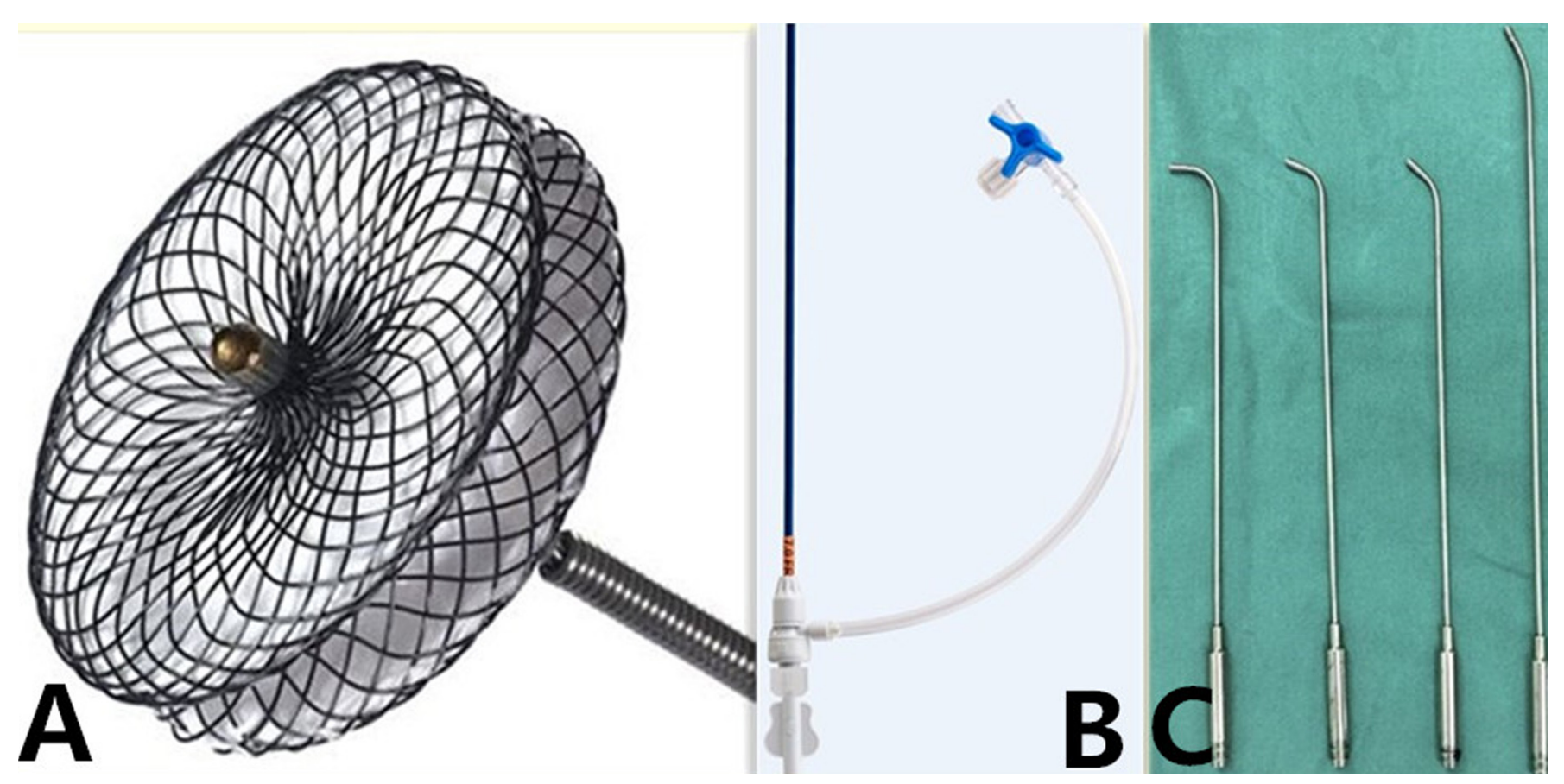

Figure 1. (A), The self-expandable symmetric occluder; (B), the delivery sheath; (C), the self-designed hollow guide steels with different tips.

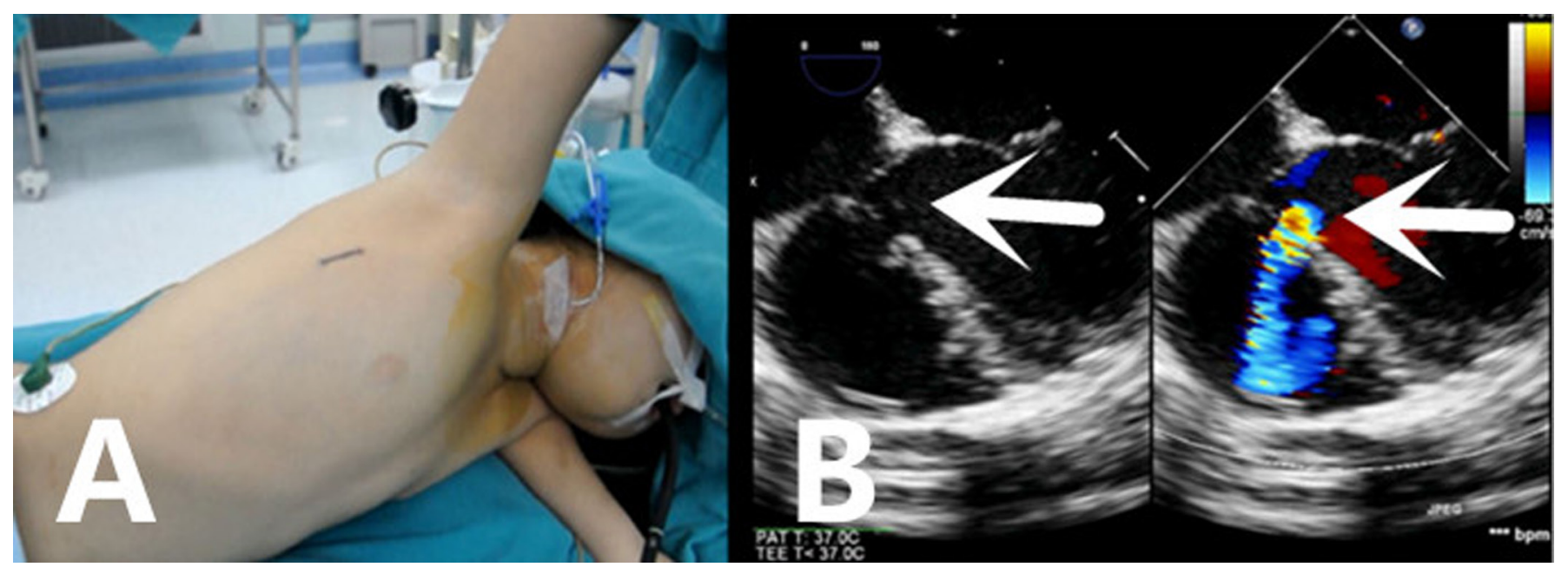

Figure 2.(A), The panel shows the patient was in left lateral position; and (B), this panel shows the echo imagings of VSD pointed to by white arrowheads.

\section{MATERIALS AND METHODS}

From January 2014 to December 2017, we retrospectively analyzed the patients diagnosed with a single pmVSD in outpatient setting undergoing transaxillary device closure of the pmVSD at Children Heart Center, Henan Provincial People's Hospital. All patients were assessed by transthoracic echocardiography (TTE; Philips iE33, Koninkliike Philips N.V., Amsterdam, The Netherlands) in outpatient setting. The echo measure was completed with the aspect of defect size and defect type with parasternal long-axis and short-axis views, apical 4-chamber view, and apical 5-chamber view. The patients can be considered candidates in this study as long as they were aged more than 6 months. The inclusion criteria includes preoperative TTE evidence of left ventricle enlargement, defect size of 3-6 mm, obvious left-to-right shunt with higher than a 3/6 systolic murmur, distance of superior rim to right aortic valve $\geq 2 \mathrm{~mm}$, TTE evidence of pulmonary artery systolic pressure less than $30 \mathrm{mmHg}$, cardiomegaly on chest $\mathrm{x}$-ray imaging, and failure to thrive according to literature. The written informed consent was obtained from patients' guardians, and the medical ethics committee of our hospital approved the study.

Patients with prolapse aortic cusp, aortic valve regurgitation, malalignment of superior and inferior rims, an amount 
Table 1. General Characteristics*

\begin{tabular}{lc}
\hline Patients $(\mathrm{N})$ & 428 \\
Age (years) (range) & $2.2 \pm 1.5(0.5-16.2)$ \\
Sex (male) & $216(51.2 \%)$ \\
Weight (kg) (range) & $8.5 \pm 4.1(6-61)$ \\
Preoperative TTE & \\
Type of VSD & Perimembranous \\
VSD diameter (mm) (range) & $4.2 \pm 1.1(3-6)$ \\
Moderate TR & $5(1.1 \%)$ \\
Severe TR & $4(0.9 \%)$ \\
\hline
\end{tabular}

*TTE, transthoracic echocardiography; VSD, ventricular septal defect; TR, tricuspid regurgitation.

Table 2. Postoperative Results*

\begin{tabular}{lc}
\hline Successful closure (n) & 422 \\
Operative time (min) (range) & $43.0 \pm 10.6(29-84)$ \\
Intracardiac time (min) (range) & $8.3 \pm 2.1(2-45)$ \\
Defects' size (mm) (range) & $4.2 \pm 1.1(3-6)$ \\
Device size (mm) (range) & $5.3 \pm 1.4(4-8)$ \\
Symmetric occluder & 437 \\
Total closure attempt(s) & 437 \\
Once (n) & 409 \\
Twice (n) & 11 \\
Thrice (n) & 2 \\
New valve regurgitation (n) & 20 \\
Mild TR (n) & 17 \\
Mild AR (n) & 3 \\
Arrhythmia & 23 \\
LAD & 1 \\
CRBBB & 16 \\
IRBBB & 6 \\
\hline
\end{tabular}

*TR, tricuspid regurgitation; AR, aortic regurgitation; LAD, left anterior fascicular block; CRBBB, complete right bundle branch block; IRBBB, incomplete right bundle branch block.

of less than $2 \mathrm{~mm}$ of superior rim to aortic valve, history of infective endocarditis, and necessity of cardiopulmonary bypass with other concomitant cardiac anomalies were excluded from enrollment.

Each patient included had the examinations of 12-lead electrocardiogram, chest $\mathrm{x}$-ray imaging, TTE, and blood test before operation.

The preoperative TTE showed moderate-to-severe tricuspid regurgitation (caused by left-to-right shunt) in 9 patients and mild aortic regurgitation (without prolapse aortic cusp) in

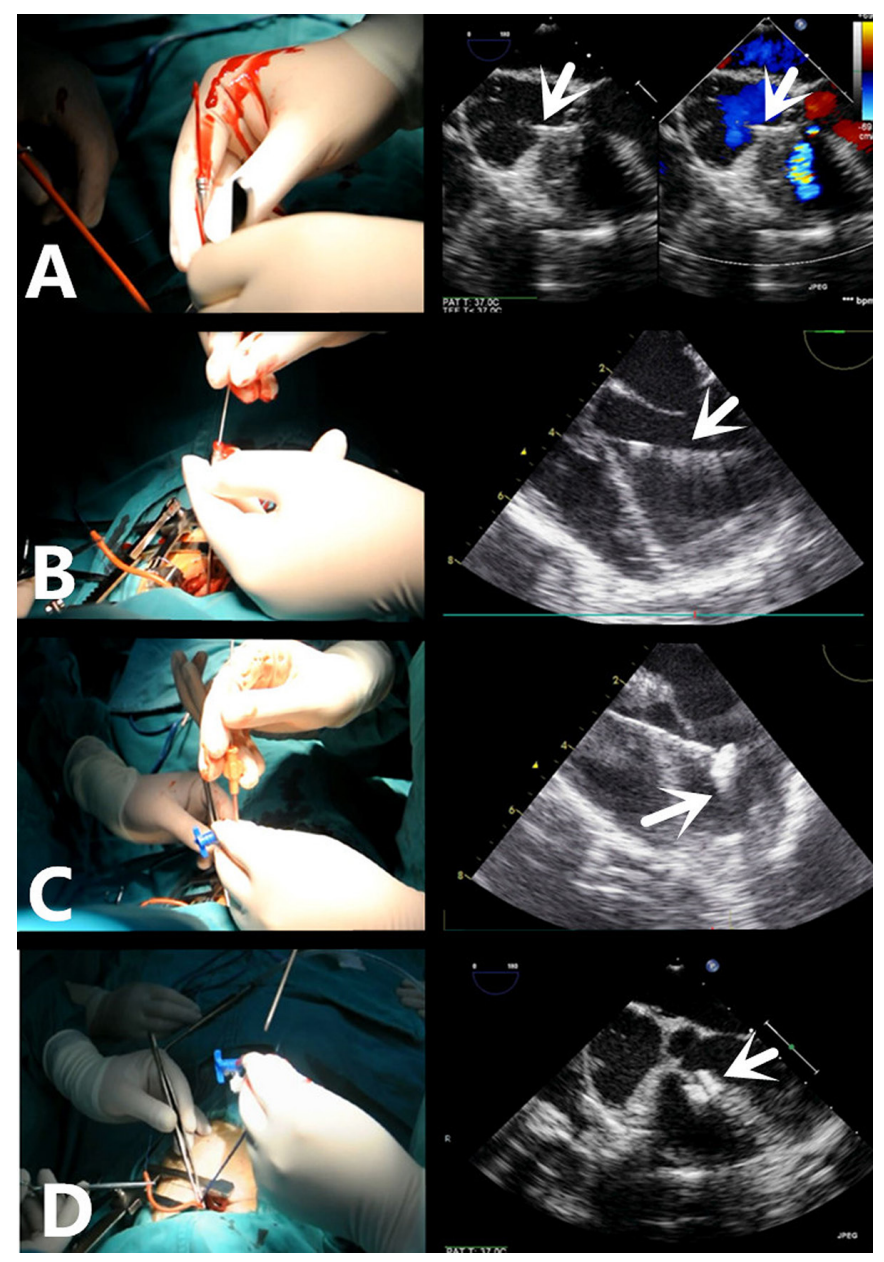

Figure 3. (A), The hollow guide steel was advanced into the right atrium, tricuspid valve, right ventricle, and VSD to build a delivery route; and the echo imagings show real-time detection of intracardiac structures. The arrowheads show the hollow steel crossing the defect. (B), The guidewire was advanced along the hollow steel to assist the delivery sheath to reach the left ventricle via the delivery route. The arrowhead shows the guidewire crossing the defect. (C), The occluder-loaded sheath was connected to the delivery sheath and open left disc under TEE guidance. The arrowhead shows the opening of the right disc of the occluder. (D), This panel shows the stability of the device in the defect after releasing by TEE. The arrowhead shows the released occluder in the defect.

2 patients. The general characteristic of all patients are shown in Table 1.

\section{Device and Delivery System}

The device and the delivery system utilized in this study are made by Lifetech Scientific (Shenzhen) Co., Ltd. (Shenzhen, China). All occluders used were symmetrical and double disc self-expandable, made of 0.005 -inch nitinol wire mesh and fabric inside. The diameter of the disc is 4-mm larger than that of the waist, and the height of the device is $3 \mathrm{~mm}$. The hollow guide steel was made by us (all are shown in Figure 1). 
Table 3. Follow-up Results*

\begin{tabular}{|c|c|c|c|c|c|c|c|c|c|}
\hline RS & - & 3 & 1 & 0 & 0 & 0 & 0 & 0 & 0 \\
\hline Mild & - & 17 & 9 & 5 & 3 & 3 & 3 & 3 & 3 \\
\hline Moderate & 5 & - & 0 & 0 & 0 & 0 & 0 & 0 & 0 \\
\hline Mild AR & 2 & 3 & 3 & 3 & 2 & 1 & 1 & 1 & 1 \\
\hline \multicolumn{10}{|l|}{ Arrhythmia } \\
\hline LAD & - & 1 & 0 & 0 & 0 & 0 & 0 & 0 & 0 \\
\hline CRBBB & - & 16 & 13 & 10 & 5 & 5 & 4 & 4 & 4 \\
\hline IRBBB & - & 6 & 4 & 3 & 3 & 3 & 3 & 3 & 2 \\
\hline
\end{tabular}

* RS, residual shunting; TR, tricuspid regurgitation; AR, aortic regurgitation; LAD, left anterior fascicular block; CRBBB, complete right bundle branch block; IRBBB, incomplete right bundle branch block; PE, pericardial effusion.

\section{Procedure}

Transaxillary device closure was performed under general anethesia in the operation room. The patient was in supine position; the transesophageal echocardiography (TEE) probe was inserted into the esophagus to confirm size and type of VSD (Figure 2). Once confirmed, the position was transferred to the left lateral position to offer a good exposure of the right armpit. According to previous TEE result, the occluder was chosen by adding $0-2 \mathrm{~mm}$ to max-VSD diameter (that is, to the maximum chosen from diameters of different echo views).

The patients were usually administrated with heparin $(1 \mathrm{mg} / \mathrm{kg})$. A 2-3 mm mini-incision between the third and fifth intercostal space was made, through which the thoracic cavity was entered. After opening the upper part of the pericardium and suspending the pericardium, the heart was well exposed. The puncture site was located $2 \mathrm{~cm}$ over the atrioventricular gap, around which the U-like suture was sewn. The hollow guide steel was advanced into the right atrium via the puncture site, then subsequently crossing the tricuspid, right ventricle, and VSD to the left ventricle to build the delivery system. The guidewire was inserted through the hollow guide steel, and then the delivery system was introduced into the left ventricle along the guidewire after retraction of the hollow steel. We selected an appropriate device and loaded it into the loading sheath after immersing it in saline to squeeze the air out. We connected the delivery sheath to the loading sheath and pushed ahead by manipulating the cable. The left-side disc was opened first, and then we retracted the device and delivery sheath simultaneously. The right-side disc was open after the left-disc reached against the ventricular septum. When the double disc was placed into the defect, the to-and-fro closure attempt was done to confirm the stability of the device (Figure $3)$. The surrounding structures, especially the aortic valve and the tricuspid valve, were checked again by TEE. The device was released and the whole delivery system was withdrawn.
When TEE showed no complications, the thoracic area was closed as usual without a drainage tube.

The patients were administrated with aspirin $(3-5 \mathrm{mg} / \mathrm{kg}$ per day) for 3-6 months immediately after operation. Physical examination, TTE, and ECG were done in outpatient setting during follow-up.

\section{Follow-up Protocol}

All patients were closely followed up at the time of 1,3 , 6, 12 months postoperatively and yearly thereafter. Adverse events were monitored in every patient by completing physical examination, TTE, and ECG.

\section{Statistics Analysis}

Data are expressed as frequency or percentage for normal variables; and mean \pm standard deviation for continuous variables; median and range, as appropriate. All statistical analyses were performed by using IBM SPSS Statistics for Windows, Version 20.0 (released 2011; IBM Corp., Armonk, NY, USA).

\section{RESULTS}

Following the inclusion criteria, a total of 422 patients (209 male) successfully underwent transaxillary occluder device closure of the pmVSD solely guided by transesophageal echocardiography. The mean age of all patients was 2.2 \pm 1.5 years (range $0.5-16.2$ years), and the mean weight was $8.5 \pm 4.1 \mathrm{~kg}$ (range 6-61 kg). During the procedure, the mean defect size measured by TEE was $4.2 \pm 1.1 \mathrm{~mm}$ (range 3-6 $\mathrm{mm}$ ), and the mean size of occluders implanted was $5.3 \pm 1.4$ (range 4-8 mm). The successful implantation of the device was achieved in 422 patients (98.6\%), whereas failure happened in 6 patients $(1.4 \%)$. All patients received an attempt to gain successful closure, except that 11 patients received 


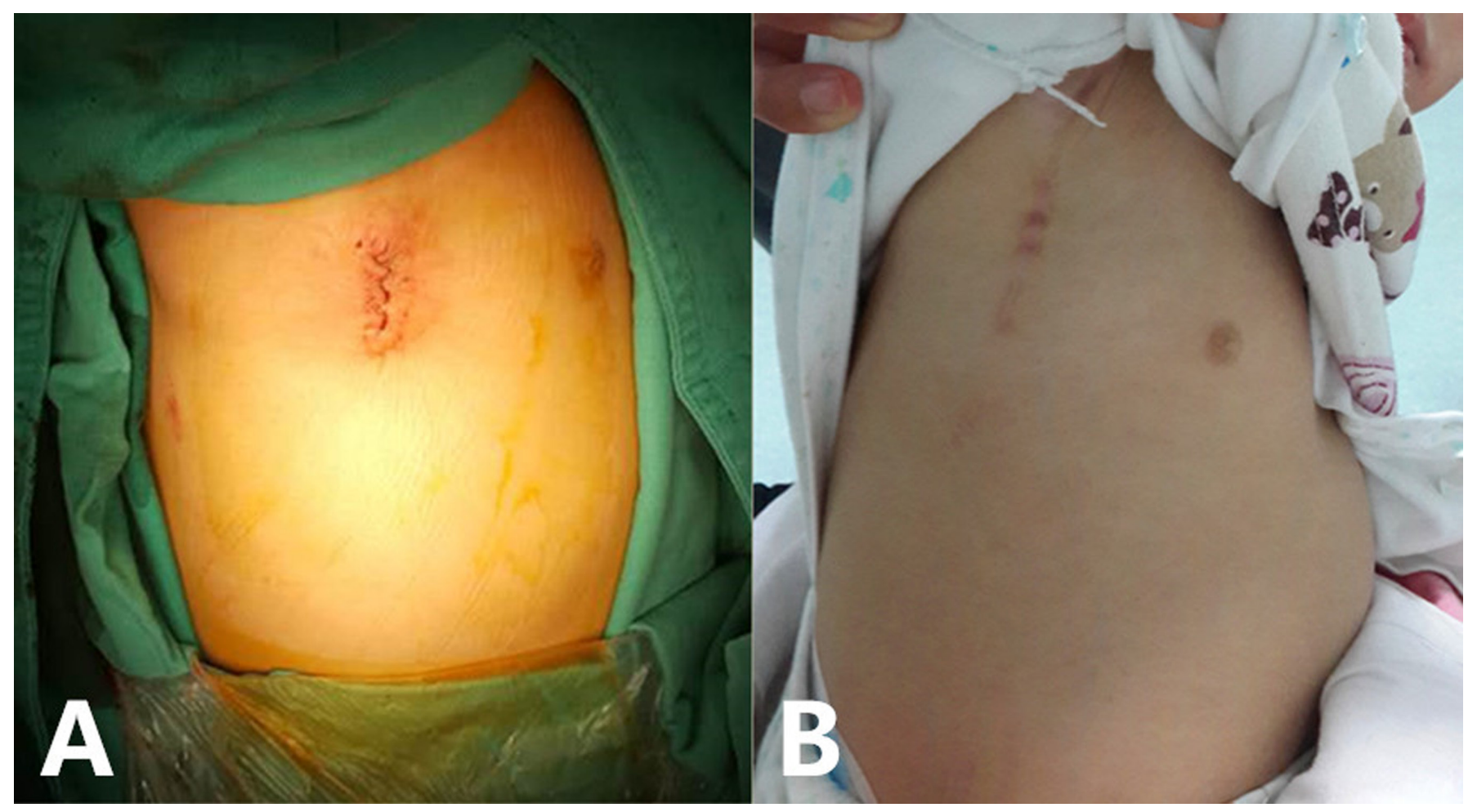

Figure 4. (A), Immediately postoperative mini-incision. (B), Follow-up mini-incision scar.

a closure attempt twice, and 2 patients received a closure attempt thrice. All preoperative moderate-to-severe tricuspid regurgitation disappeared. (Details are shown in Table 2.)

\section{Early Adverse Event Postoperatively}

In $422(98.6 \%)$ successful procedures, early adverse events emerged in 47 patients $(11.1 \%)$, including left anterior block, complete right bundle branch block (CRBBB), incomplete right bundle branch block (IRBBB), and new aortic and tricuspid valve regurgitation.

The moment after the device was released, new mild tricuspid regurgitation was detected by TEE in 17 patients, and mild aortic regurgitation in 3 patients. No complete AVB occurred, except for several arrhythmia events, including LAD in one patient, CRBBB in 16 patients, and IRBBB in 6 patients; and all of them were administrated with close follow-up. A trivial residual shunt was detected in 3 patients, $1 \mathrm{~mm}, 1.5 \mathrm{~mm}, 1 \mathrm{~mm}$, respectively, and was managed with close follow-up.

Pericardial effusion was found in 2 patients when they were transported to the general ward after extubation, and it was solved with tube drainage.

\section{Conversion to Surgical Repair}

Six patients $(1.4 \%)$ failed to have device closure of the pmVSD. The reasons resulting in failure included new aortic valve regurgitation, inappropriate deployment, and severe resident shunt. Device-related aortic regurgitation appeared with an opening left disc impinging on the right aortic valve in 3 patients. In 3 patients, the left-to-right shunt showed no significant decrease by TEE, the defect of which was found irregular and oval; and the longest diameter could not be measured by TTE and TEE. The final patient of these 6 underwent device closure smoothly, but TTE in ICU showed the device had a big range of mobilization; the patient was transported with emergency to the operation room, and the device was proved dislocated. All 6 patients underwent open heart surgery uneventfully.

\section{Follow-up Results}

The follow-up was totally available for 408 patients (96.7\%), whereas 4 patients missed the follow-up. The incision for this technique is minimal and can appear hidden in the armpit invisibly during follow-up (Figure 4). The mean duration of follow-up was 36.8 months (range 6-60 months). No mortality and major adverse events were noted. Patients with frequent respiration infection had no recurrence; patients with failure to thrive had recovered in growth matched with development criteria; cardiomegaly resolved in all the followup patients.

During follow-up, the patients with arrhythmia had no aggravation. The patient with previous LAD recovered to healthy status; patients with CRBBB and IRBBB had no change without any relevant symptom. The mild tricuspid valve regurgitation in 17 patients disappeared except for 5 cases until the latest follow-up. Among 3 previous mild aortic valve regurgitation cases, 2 cases disappeared at 3rd-month follow-up, and the other one had no progress towards aggravation. All 3 patients with trivial residual shunt showed a complete closure at 3rd-month and 6th-month follow-ups. The 
2 patients with pleural effusion before discharge had no recurrence by TTE at follow-up (details are shown in Table 3).

\section{DISCUSSION}

Traditional surgical repair on $\mathrm{CPB}$ has been considered the optimal choice for closure of the pmVSD, with excellent results, but simultaneously with risks of $\mathrm{CPB}$, great trauma of midline sternotomy, and transfusion, each of which could pose a negative effect on postoperative recovery [Xing 2009; Song 2017]. Percutaneous transcatheter closure through use of fluoroscopy avoids $\mathrm{CPB}$, great trauma, and transfusion; however, this technique needs expensive devices, which is not only impossible in a third world country, but also brings radiation damage to performers and patients. Besides, the advance of the guidewire and delivery sheath in vessels is invisible and could only be located by imagings of fluoroscopy, causing injuries to vessel endothelium. The transthoracic surgical device closure, combining merits of surgery and percutaneous transcatheter, has achieved excellent closure results since its introduction [Xing 2009; Song 2017].

Transaxillary occluder device closure is performed via a right infra-axillary minimal incision, on the beating heart, under the guidance of TEE, avoiding CPB, great trauma, and radiation. Inferior median sternotomy is a common incision for transthoracic closure technique [Xing 2009; Xing 2010]. Though this incision has less trauma than median sternotomy, an obvious scar still is left on the chest wall. Different from inferior median sternotomy, the transaxillary approach adopts the infra-axillary minimal incision, the postoperative scar of which is hidden in the armpit, bringing both minimal effect and cosmetical aesthetics.

In this study, the use of the cavity bougie confirms the feasibility of the building of the delivery route. The technique, which goes from the right atrium to the left ventricle subsequently through the tricuspid valve and VSD, the route of which is tortuous, not straight, is different from traditional transthoracic technique with puncture of right free ventricle. In addition, the introduction of device deployment into the intracardiac structure from the right atrium avoids the injury of the right ventricular muscle and coronary arteries, which may be at risk of right ventricle dysfunction.

By reviewing previous literatures, we know that AVB is an extremely adverse event in all postoperative complications [Bai 2015]. We have not encountered such an adverse event of AVB from the beginning until the latest follow-up. Published reports show that the high incidence of occurrence was closely associated with transcatheter device closure, where a long loop delivery route and oversized and inflexible occluders were used, leading to compressure around the defect rim with radial force, especially on the conduction bunch running beneath the inferior rim of the defect [Xing 2015]. The occluders in this study were modified with more flexible materials, and generally the size of device was selected appropriately, ameliorating the compressure effect on defects' rims, which avoided interference with the conduction bunch going along the inferior rim of the defect; therefore, the AVB event is rare in transaxillary device closures. The total ECG events are CRBBB, and IRBBB, among which happened in only one patient and was relatively life-threatening. Fortunately, LAD disappeared at discharge.

Valve regurgitation is also a common complication postoperatively. Nine patients in this study with moderate-to-severe tricuspid regurgitation were included. Preoperative TTE showed that all tricuspid regurgitations of these patients were induced by left-to-right shunt, not caused by dysfunction of tricuspid valve. The operative result demonstrated that with a disappearance of tricuspid regurgitation. Although 17 mild tricuspid regurgitation cases were newly detected, the tricuspid regurgitation gradually disappeared during follow-up. Two mild preoperative aortic regurgitation cases, probably due to a hit on the aortic valve due to left-to-right shunt, disappeared after successful closure. Three new mild aortic regurgitation cases occurred, and we postulated that slight impinging on the right aortic leaflet contributed to this phenomenon. For them, we very closely followed them with respect to aortic regurgitation by TTE and found 2 patients relieved from aortic regurgitation, though one case remained until the latest follow-up. Therefore, we concluded that slight impinging could disappear with persistent mobility and form a relatively little interference between the left disc and the right aortic leaflet.

\section{CONCLUSION}

In our experience, the incidence of a serious adverse event is very low and has a successful rate, no lower than previous reports, confirming that the transaxillary occluder device closure of the pmVSD is a valuable alternative treatment in strictly selected pmVSD with promising early and midterm outcomes. Potential later onset conduction occurrence remains a common concern, so further follow-up study is needed to prove the latent risk factor for this technique.

\section{ACKNOWLEDGMENT}

The authors identify that there are not any conflicts of interest among the authors.

\section{REFERENCES}

Bai Y, Xu XD, Li CY, et al. 2015. Complete atrioventricular block after percutaneous device closure of perimembranous ventricular septal defect: a single-center experience on 1046 cases. Heart Rhythm 12(10):2132-40.

Bouma BJ, Mulder BJ. 2017. Changing landscape of congenital heart disease. Circ Res 120(6):908-22.

Hufnagel CA, Conrad PW. 1965. Transaortic repair of ventricular septal defects. Am J Surg 110:448-51.

Hong ZN, Chen Q, Lin ZW, et al. 2018. Surgical repair via submammary thoracotomy, right axillary thoracotomy and median sternotomy for ventricular septal defects. J Cardiothorac Surg 13(1):47.

Ghosh S, Sridhar A, Sivaprakasam M. 2018. Complete heart block 
following transcatheter closure of perimembranous VSD using amplatzer duct occluder II. Catheter Cardiovasc Interv 92(5):921-4.

Green L, Oakley CM, Davies DM, Cleland WP. 1965. Successful repair of left ventricular aneurysm and ventricular septal defect after indirect injury. Lancet 2(7420):984-6.

Karaçelik M, Öztürk P, Doyurgan O, et al. 2015. A complication following the transcatheter closure of a muscular ventricular septal defect. J Tehran Heart Cent 10(3):149-51.

Narin N, Pamukcu O, Tuncay A, et al. 2018. Percutaneous ventricular septal defect closure in patients under 1 year of age. Pediatr Cardiol 39(5):1009-15.

Ou-Yang WB, Li SJ, Wang SZ, et al. 2015. Echocardiographic guided closure of perimembranous ventricular septal defects. Ann Thorac Surg 100(4):1398-402.

Patel NH, Madan TH, Panchal AM, Thakkar BM. 2009. Percutaneous closure of patent ductus arteriosus via internal jugular vein in patient with interrupted inferior vena cava. Ann Pediatr Cardiol 2(2):162-4.

Polat TB, Türkmen E. 2016. Transcatheter closure of ventricular septal defects using the Amplatzer Duct Occluder II device: a single-center experience. Postepy Kardiol Interwencyjnej 12(4):340-7.

Song S, Fan T, Li B, et al. 2017. Minimally invasive peratrial device closure of perimembranous ventricular septal defect through a right infraaxillary route: clinical experience and preliminary results. Ann Thorac Surg 103(1):199-204

Song X, Xing Q, Wu Q, Ren Y, Wan H, Li J. 2017. Transthoracic device closure of juxtaarterial ventricular septal defects: midterm follow-up results. Ann Thorac Surg 104(3):841-6.

Xing Q, Pan S, An Q, et al. 2010. Minimally invasive perventricular device closure of perimembranous ventricular septal defect without cardiopulmonary bypass: multicenter experience and mid-term follow-up. J Thorac Cardiovasc Surg 139(6):1409-15.

Xing Q, Wu Q, Shi L, Xing Y, Yu G. 2015. Minimally invasive transthoracic device closure of isolated ventricular septal defects without cardiopulmonary bypass: long-term follow-up results. J Thorac Cardiovasc Surg 149(1):257-64.

Xing QS, Pan SL, Zhuang ZY, et al. 2009. Minimally invasive perventricular device closure of an isolated perimembranous ventricular septal defect with a newly designed delivery system: preliminary experience. J Thorac Cardiovasc Surg 137(3):556-559. 